\title{
Enquête sur les pratiques de surveillance et de vaccination relatives à la coqueluche au Canada, 2012
}

\author{
Desai $\mathrm{S}^{1^{*}}$, Smith $\mathrm{T}^{1}$ et Deehan $\mathrm{H}^{1}$
}

1. Agence de la santé publique du Canada, Centre de l'immunisation et des maladies respiratoires infectieuses, Maladies évitables par la vaccination, Ottawa (ON)

* $\quad$ Auteure-ressource : shalini.desai@phac-aspc.gc.ca

\section{Résumé}

Contexte : En 2012, on a observé une hausse de l'incidence de la coqueluche dans les Amériques. L'Organisation panaméricaine de la santé (OPS) a émis un certain nombre de recommandations en vue de renforcer la surveillance, d'enquêter sur les éclosions, d'évaluer le bien-fondé et l'opportunité de la vaccination dans la population et de surveiller les politiques relatives aux personnes présentant un risque élevé, comme les travailleurs de la santé et les femmes enceintes.

Objectif : Examiner les mesures prises par les provinces et les territoires du Canada en vue de prévenir la propagation de la coqueluche.

Méthodes : Une enquête élaborée à partir des recommandations de l'OPS a été envoyée à tous les territoires/provinces par l'intermédiaire du Conseil des médecins hygiénistes en chef.

Résultats : Toutes les provinces et les territoires ont participé à l'enquête. L'utilisation de définitions uniformes de cas favorise une surveillance rigoureuse; la plupart des provinces et les territoires utilisent la définition nationale d'un cas. Les éclosions sont l'objet d'investigations à l'échelon local ou provincial. La couverture vaccinale n'est pas bien connue, mais on s'efforce d'améliorer la surveillance au moyen d'enquêtes et de registres de vaccination. Des politiques relatives aux personnes présentant un risque élevé ont été mises en œuvre, mais n'ont pas encore fait l'objet d'évaluations.

Conclusion : Selon les recommandations de I'OPS, le Canada est en bonne position pour fournir des données de surveillance concernant la coqueluche. Des lacunes relatives à la surveillance, à la standardisation des pratiques entre les administrations et aux données concernant la couverture vaccinale peuvent devoir être corrigées afin que l'on arrive à une meilleure compréhension des répercussions de la coqueluche au Canada.

\section{Introduction}

La coqueluche est une maladie évitable par la vaccination causée par la bactérie Bordetella pertussis.

L'épidémiologie de la coqueluche est marquée par une apparition cyclique de la maladie, qui se manifeste environ tous les deux à cinq ans. Au Canada, un programme de vaccination existe depuis 1943. Depuis la mise en œuvre de ce programme, on a constaté une réduction spectaculaire de l'incidence de la maladie. Avant sa mise en œuvre, le taux d'incidence avait atteint un sommet de 181 cas pour 100000 personnes ${ }^{1}$. Depuis, il a décliné, pour atteindre en 2011 la plus faible incidence jamais enregistrée de 2 cas pour 100000 personnes ${ }^{2}$. En 2012, au Canada, l'incidence de la maladie a été multipliée par sept comparativement à l'année précédente. De plus, on a également constaté une hausse de l'activité de la coqueluche dans la région des Amériques. En réponse à cet accroissement, l'Organisation panaméricaine de la santé (OPS) ont annoncé des recommandations sur la surveillance et l'immunisation contre la coqueluche). 


\section{Recommandations de I'OPS sur la surveillance et l'immunisation contre la coqueluche ${ }^{3}$}

1. Renforcer la surveillance afin de surveiller le fardeau de la maladie, d'évaluer l'incidence de l'immunisation au moyen de la vaccination et de repérer les éclosions. Chaque éclosion de coqueluche devrait être rigoureusement étudiée - cela permettrait de mieux comprendre l'épidémiologie actuelle de la maladie dans la région des Amériques.

2. Analyser la couverture vaccinale des nourrissons de 1 an et des enfants de moins de 5 ans, en mettant particulièrement l'accent sur la détermination des groupes sans couverture vaccinale.

3. Les pays doivent s'assurer que la couverture vaccinale est $=95 \%$ avec trois doses d'un vaccin anticoquelucheux chez les enfants de moins de 1 an et encourager la vaccination et la réalisation du calendrier en temps opportun. La quatrième dose du vaccin diphtérie-tétanos-coqueluche (DTC) devrait être intégrée au programme régulier de vaccination de tous les pays et la couverture obtenue grâce à cette dose (comme dans le cas de toutes les doses de vaccin) doit faire l'objet d'une consignation, d'un suivi, d'une déclaration et d'une évaluation rigoureux.

4. Vacciner les travailleurs de la santé pour éviter la transmission en milieu hospitalier aux nourrissons de moins de 6 mois et aux personnes dont le système immunitaire est affaibli.

5. Immuniser les femmes enceintes lors d'une éclosion pour mieux protéger les nouveau-nés.

Le présent article vise à examiner les mesures prises par les administrations au Canada en réponse à l'augmentation de l'activité de la coqueluche, ainsi qu'à évaluer les forces et les défis du Canada relativement aux recommandations de l'OPS.

\section{Méthodes}

À l'aide de Fluid Surveys, l'Agence de la santé publique du Canada, en collaboration avec les responsables de la santé publique en Ontario, a élaboré et réalisé une enquête électronique. La validité apparente et la validité de contenu de cette enquête ont été évaluées par trois réviseurs. L'enquête comportait 35 questions concernant les priorités relatives à la surveillance de la coqueluche établies par l'OPS. La première partie (16 questions) était axée sur la surveillance de la maladie, les définitions de cas et les pratiques en matière de déclaration. La seconde partie (19 questions) portait sur les pratiques de vaccination, la couverture vaccinale et l'évaluation des programmes. On a demandé au médecin hygiéniste en chef de chaque province/territoire de répondre au questionnaire. Le questionnaire est disponible sur demande auprès de l'auteur correspondant.

Les enquêtes remplies ont été regroupées et analysées en fonction de thèmes communs. On a effectué une analyse descriptive des réponses.

\section{Résultats}

Le taux de réponse à l'enquête était de $100 \%$ et le taux de données complètes, de $92 \%$. Un territoire n'a pu remplir l'enquête dans son intégralité.

\section{Surveillance}

Toutes les administrations, à l'exception de trois, utilisent la définition nationale de cas; deux d'entre elles utilisent une définition moins précise qui ne requiert pas la présence de symptômes cliniques à la suite de la détection de I'ADN de B. pertussis dans un échantillon clinique. Une province exige la présence de symptômes cliniques en 
plus de la confirmation en laboratoire, peu importe la méthode de confirmation (c.-à-d. isolement ou détection de I'ADN de B. pertussis); ainsi, sa définition d'un cas est plus précise que la définition nationale.

Pour chacune des administrations, la déclaration se fait à divers moments du cycle. Une province reçoit les déclarations presque en temps réel, les cas probables aussi bien que confirmés étant déclarés toutes les heures, alors que d'autres administrations ont des calendriers de déclaration quotidiens, hebdomadaires ou mensuels. Les données recueillies varient également. Tous les territoires/provinces recueillent des renseignements concernant l'âge, le sexe, le lieu de résidence ainsi que la méthode et les résultats de l'analyse de laboratoire. De nombreuses administrations ont la capacité d'obtenir d'autres renseignements, grâce à des investigations des cas menées à l'échelle locale; deux administrations peuvent aussi obtenir des renseignements à propos des antécédents de vaccination par des sources de données distinctes des données de surveillance, comme des registres de vaccination ou des banques de données d'agences de santé publique sur la vaccination. Fait intéressant, tous les territoires/provinces ne recueillent pas systématiquement les renseignements concernant les antécédents de vaccination; pourtant, une telle collecte contribuerait à comprendre s'il y a eu échec de l'immunisation ou absence de vaccination. Parmi les autres renseignements qui ne sont pas uniformément recueillis par les provinces/territoires, citons les symptômes, les complications, la durée de l'hospitalisation et l'issue de la maladie.

\section{Enquête sur les éclosions}

En présence d'une éclosion, la plupart des administrations accroissent leur surveillance de la coqueluche pour inclure une modification de la définition d'un cas, des types de variables recueillies et de la façon dont les données sont recueillies. Les modifications apportées à la définition de cas sont en fonction des caractéristiques de l'éclosion. Dans certains territoires/provinces, on recueille plus de données lors d'une éclosion, notamment sur le contexte de transmission, les contacts, les facteurs de risque, les soins préventifs, ainsi que des données plus précises sur les analyses de laboratoire.

À la lumière des éclosions, nombre de provinces/territoires ont récemment modifié leur surveillance de la coqueluche en vue de mieux comprendre le fardeau de la maladie ou d'évaluer les effets de leurs programmes d'immunisation. Ces changements comprennent des analyses de laboratoire supplémentaires, l'ajout de variables pour étudier la transmission, en particulier parmi les nourrissons et les membres de leur famille, l'accroissement de l'importance accordée aux antécédents de vaccination afin de déterminer l'efficacité des programmes d'immunisation, l'utilisation de logiciels supplémentaires pour comprendre les variations de l'incidence et l'amélioration de la communication avec les fournisseurs de première ligne. Certaines provinces et territoires ont également augmenté la fréquence de leurs analyses des données de surveillance systématique en vue d'améliorer leur capacité à répondre à la hausse de l'activité de la maladie.

\section{Couverture vaccinale}

\section{Enfance}

L'ensemble des provinces/territoires incluent un vaccin anticoquelucheux dans leur calendrier d'immunisation systématique des enfants. Les cibles de couverture vaccinale ont été définies à l'échelon national ${ }^{4}$. Parmi les administrations qui ont répondu aux questions relatives aux cibles de vaccination, quatre d'entre elles ont utilisé les cibles nationales afin d'évaluer leurs programmes d'immunisation, trois ont des cibles distinctives et cinq ont dit ne pas avoir établi de cibles. Malgré cette absence d'uniformité en matière de cibles, l'ensemble des provinces/territoires ont recours à des méthodes pour tenter d'améliorer leur couverture vaccinale. Une seule province envoie directement un avis automatique généré par ordinateur aux familles dont un enfant atteint l'âge de sept ans. Les évaluations de la couverture se font par enquêtes, données de registres de vaccination ou carnets de vaccination scolaire, mais bon nombre d'administrations comptent sur les vaccinateurs pour les aider à respecter le calendrier et à tenir à jour les dossiers de vaccination.

Étant donné le rôle primordial que jouent les fournisseurs de première ligne dans la couverture vaccinale, l'ensemble des provinces/territoires utilisent diverses méthodes pour communiquer et diffuser l'information qui 
aiderait les fournisseurs, notamment la publication régulière de rapports sur la couverture et l'affichage direct des estimations de la couverture vaccinale sur la page Web de santé publique de l'administration en question.

\section{Immunisation des adultes et personnes présentant un risque élevé}

Les programmes d'immunisation des adultes contre la coqueluche sont financés par l'État dans sept administrations, mais la plupart requièrent que l'adulte prouve qu'il n'a jamais reçu de vaccin pour être admissible à la vaccination. L'immunisation des travailleurs de la santé est recommandée par la plupart des administrations.

Trois administrations ont recommandé l'immunisation des femmes enceintes, en raison de l'augmentation de l'activité de la coqueluche. La plupart des autres administrations recommandent l'immunisation des mères en fonction de l'épidémiologie locale et de la consultation avec un fournisseur de soins de santé. Quelques administrations ont recours à la vaccination des personnes en contact étroit avec un nouveau-né, ou au " coconnage », comme méthode d'intervention. L'immunisation maternelle et le coconnage n'ont pas été officiellement évalués au Canada.

\section{Analyse}

L'OPS a présenté à chaque État membre une liste de cinq recommandations en réponse à une hausse de l'activité de la coqueluche. En comparant cette liste et les activités entreprises par les différentes administrations au Canada, on constate que notre système de surveillance et de réponse fournit bon nombre des éléments recommandés par l'OPS, mais que des mesures supplémentaires peuvent être prises afin d'améliorer notre capacité à décrire l'activité et les éclosions de la maladie, de parfaire notre compréhension de la couverture vaccinale et de protéger les groupes vulnérables.

L'OPS recommande de renforcer la surveillance en vue de surveiller le fardeau de la maladie, de détecter les éclosions et d'évaluer les effets des programmes d'immunisation. Au Canada, la coqueluche fait partie des maladies à déclaration obligatoire à l'échelle nationale, mais notre capacité à évaluer les effets de la vaccination et à détecter les éclosions est limitée par le calendrier actuel de déclaration à l'échelle nationale (une fois l'an) et par le format dans lequel les données sont reçues (agrégées uniquement). À l'échelon provincial/territorial, la coqueluche est surveillée systématiquement et beaucoup plus en détail. Les systèmes des provinces/territoires ont permis de détecter des éclosions. La surveillance systématique varie d'une administration à l'autre, en ce qui concerne non seulement les calendriers, mais aussi les données recueillies. Si de plus amples renseignements sont requis à l'échelon national, il est possible d'adresser une demande de données aux provinces/territoires. Afin de renforcer notre système actuel, il s'agirait d'adopter des méthodes telles qu'une déclaration plus détaillée et opportune à l'échelon national et une capacité améliorée de consignation des antécédents de vaccination de chaque cas ainsi que de la population en général.

La capacité de mener une enquête rigoureuse sur une éclosion n'existe pas dans le cadre du programme national de surveillance. Des enquêtes approfondies se produisent à l'échelle locale, provinciale et territoriale partout au Canada. On peut obtenir de l'aide en vue d'enquêter sur une éclosion par l'intermédiaire de programmes nationaux tels que le Programme canadien d'épidémiologie de terrain. À la survenue d'une éclosion, les administrations peuvent communiquer de nombreuses façons, par exemple au moyen d'alertes de santé publique affichées en passant par le Réseau canadien de renseignements sur la santé publique, par des communautés de groupes d'experts du Réseau pancanadien de santé publique, comme le Comité canadien d'immunisation, et par des organismes de haute instance, comme le Conseil des médecins hygiénistes en chef. La capacité de diffuser des observations concernant les éclosions est limitée par les ressources. Un certain nombre d'administrations ont rédigé et largement diffusé leurs observations tirées de leurs expériences face à des éclosions, mais ce ne sont pas toutes les administrations qui disposent de cette capacité. Cette situation nuit à notre pouvoir d'apprendre de nos expériences collectives.

Le Canada réalise tous les deux ans une enquête nationale sur la vaccination, mais la taille de l'échantillon ne permet pas généralement de connaître les variations d'une administration à l'autre, ni d'analyser des groupes 
précis qui pourraient être sous-vaccinés ou ne pas l'être du tout. De nombreuses administrations se tournent vers les registres de vaccination et ont (ou auront) ainsi la possibilité d'analyser la couverture vaccinale et de générer des rappels destinés aux personnes qui ne sont pas à jour. Les défis de la couverture vaccinale consistent à s'assurer que l'ensemble des provinces/territoires du Canada ont cette possibilité et que les méthodes utilisées pour obtenir les estimations de la vaccination sont comparables.

L'OPS a également recommandé que tous les travailleurs de la santé soient immunisés contre la coqueluche, ainsi que les femmes enceintes, en situation d'éclosion. Au Canada, les administrations sont appuyées par les recommandations du Comité consultatif national de l'immunisation (CCNI), qui comprennent l'immunisation des

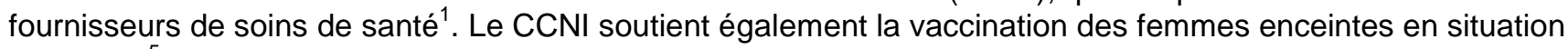
d'éclosion 5 .

En fonction des recommandations de l'OPS et de l'obligation qui incombe au Canada en tant que membre de la communauté internationale, la présente enquête a d'importantes incidences sur la pratique. Dans le cas d'un fournisseur de première ligne, la vigilance continue, les analyses appropriées et la déclaration des personnes qui présentent des symptômes compatibles avec la coqueluche demeurent de la plus haute importance. Les déclarations de cas sont à la base d'une surveillance rigoureuse et permettent la détection des éclosions nécessitant le type d'investigations exhaustives qui peuvent améliorer notre compréhension de la maladie. Comme chaque ordre de gouvernement joue un rôle dans la surveillance, la couverture vaccinale et la protection des groupes vulnérables, la présente enquête souligne aussi les occasions d'amélioration du point de vue plus vaste de la santé publique. Par exemple, la compréhension du type de données recueillies à l'échelle provinciale/territoriale et des différences qui existent entre ces collectes orientera les délibérations à l'échelle nationale à propos de la surveillance future de la coqueluche. En outre, une analyse et une évaluation approfondies relatives aux changements que diverses administrations ont mis en œuvre en matière de surveillance et de mesures de santé publique peuvent déboucher sur la détermination de pratiques exemplaires fondées sur des données probantes concernant la lutte contre la coqueluche au Canada.

\section{Conclusions}

Le Canada surveille systématiquement la coqueluche et peut fournir une perspective nationale sur l'incidence de la maladie, ainsi qu'un tableau épidémiologique plus détaillé au moyen des données de surveillance provinciales/territoriales, s'il y a lieu. II maintient des cibles de couverture vaccinale nationale qui correspondent aux recommandations de l'Organisation panaméricaine de la santé. II évalue systématiquement la couverture vaccinale générale au moyen d'enquêtes menées auprès de la population et possède des recommandations nationales visant l'immunisation des travailleurs de la santé et des femmes enceintes dans des situations d'éclosion. Les lacunes actuelles dans les pratiques de surveillance et d'immunisation canadiennes sont la disponibilité d'une information détaillée sur l'activité de la coqueluche à l'échelle nationale. En outre, l'absence de données d'immunisation facilement disponibles sur les cas et la population en général limite notre compréhension de l'incidence de l'immunisation sur le fardeau de la maladie et empêche une évaluation valable des programmes d'immunisation au Canada. Ces problèmes ont été soulevés auparavant et font l'objet d'une analyse dans le cadre de l'élaboration des ententes sur l'échange de renseignements avec les partenaires provinciaux/territoriaux et du renouvellement de la Stratégie nationale d'immunisation.

\section{Références}

1. Agence de la santé publique du Canada. Guide canadien d'immunisation (G.C.I.). Édition évolutive. Ottawa. Partie 4 - Vaccins actifs : Vaccin contre la coqueluche. Accès : http://www.phac-aspc.gc.ca/publicat/cig-gci/p04-pert-coqu-fra.php

2. Smith T., Rotondo J., Desai S., Deehan H. « Surveillance de la coqueluche au Canada: tendances jusqu'à 2012 ». RMTC 2014;40S3:19-29

http://webqa.phac-aspc.gc.ca/publicat/ccdr-rmtc/14vol40/dr-rm40-03/dr-rm40-03-per-fra.php 
3. Organisation panaméricaine de la santé. Epidemiological Alert: Pertussis (Whooping Cough), (16 novembre 2012).

http://www.paho.org/hq/index.php?option=com_docman\&task=doc_view\&gid=19323\&ltemid=\&lang=en ; [disponible en anglais seulement].

4. Agence de la santé publique du Canada. « Rapport final sur les résultats de la Conférence nationale de concertation sur les maladies évitables par la vaccination au Canada ». RMTC 2008;34S2:1-56 http://www.phac-aspc.gc.ca/publicat/ccdr-rmtc/08vol34/34s2/index-fra.php

5. CCNI. Vaccination contre la coqueluche durant la grossesse.

\section{Remerciements}

Les auteures souhaitent remercier les membres du Conseil des médecins hygiénistes en chef et leurs équipes des provinces/territoires d'avoir répondu à cette enquête, ainsi que la province de l'Ontario d'avoir offert son aide dans l'élaboration de l'outil d'enquête.

\section{Conflit d'intérêts}

Aucun conflit à déclarer.

\section{Source de financement}

Aucun. 